\title{
El Programa Pueblos Mágicos Y ALGUNOS DE SUS EFECTOS LOCALES
}

MANUELA GUILLÉN LÚGIGO manuela.guillen@unison.mx Universidad de Sonora

En esta nota se plasma una semblanza general del Programa Pueblos Mágicos y de algunos de sus efectos, con base en las evidencias empíricas de que se dispone producto de investigaciones en las que la autora de este ensayo ha colaborado y de reflexiones con colegas participantes en las mismas, en diferentes localidades en las que ha operado el programa en mención. Se parte de la presentación de algunas de sus características más relevantes, para centrarse posteriormente en la identificación de lo que se consideran como sus luces y sombras, tomando en cuenta algunos de sus resultados más destacados. Se concluye que el ángulo positivo del programa se centra en los beneficios operados en la infraestructura y fisonomía de los pueblos, así como en las diversificaciones parcialmente operadas en los giros económicos con el fortalecimiento del comercio y los servicios; sin embargo, estos no se han reflejado hasta ahora en beneficios para la población en su conjunto.

Palabras clave: Pueblos mágicos, luces, sombras, programa, beneficios.

\section{The Magical Towns Program and some of ITS local effects}

This essay contains a general profile of the Magical Towns Program and some of its effects, based on the empirical evidence available as a result of research in which the author of this work has collaborated and reflections with colleagues participating, in different localities where the program in question has operated. It begins with the presentation of some of its most relevant characteristics, to later focus on the identification of what are considered as its lights and shadows, taking into account some of its most outstanding results. It is concluded that the positive angle of the program is centered in the benefits operated in the infrastructure and physiognomy of the towns, as well as in the diversifications partially operated in the economic sectors with the strengthening of trade and services; however, these have not been reflected so far in benefits for the population as a whole.

Keywords: Magical Towns, lights, shadows, program, benefits.

Fecha de recepción: 15 diciembre de 2020

Fecha de aceptación: 14 de enero de 2021

CÓMO CITAR: Guilén, M. (2021). El programa Pueblos Mágicos y algunos de sus efectos locales. Dimensiones Turísticas 5(8), 131-141. https://doi.org/10.47557/MJCU3028 


\section{Introducción}

I I turismo se ha convertido en uno de los sectores más importantes de la economía, tanto en el plano global como a nivel nacional. En México, la denominada "industria sin chimeneas" constituye hoy por hoy una de las actividades más destacadas, tanto por sus aportaciones al PIB como por la derrama económica que genera, derivada de la creciente afluencia de turistas internacionales y nacionales a los distintos sitios y localidades regionales que poseen atractivos naturales, históricos, culturales, entre otros.

Según datos oficiales, México ocupó en 2016 el octavo lugar mundial por número de llegadas de visitantes y el decimocuarto en ingresos de divisas generados con la actividad turística (Secretaría de Turismo, Sectur, 2017). Además, se ha afirmado que el turismo representa el $8.7 \%$ del Producto Interno Bruto (PIB) nacional y que ha generado aproximadamente 2.3 millones de puestos de trabajo (Instituto Nacional de Estadística y Geografía, INEGI, 2019). Esta es la perspectiva alentadora del turismo, a pesar de la reciente retracción del sector y reducción de los flujos turísticos a nivel mundial, como efecto de los confinamientos por la pandemia de COVID-19.

Regularmente el turismo ha sido reconocido como detonante de la actividad económica, merced a las derramas derivadas de las empresas y servicios turísticos e incluso como alternativa para la reactivación económica de localidades que han visto agotadas o en peligro sus fuentes principales de ingresos, como es el caso de las actividades primarias, por ejemplo. Pero la otra cara de la moneda está configurada por los diversos efectos negativos del turismo en los entornos donde este tiene lugar, o por el favor selectivo de los planes, programas y las propias empresas turísticas, que benefician a segmentos reducidos de la población local.

El denominado turismo cultural ha tenido visibilidad y relevancia en México en los últimos 20 años. La promoción de este tipo de turismo se presenta a través del Programa Pueblos Mágicos (PPM), cuyo propósito central ha sido favorecer el desarrollo de las localidades donde se pone en marcha. Se trata de comunidades con determinados atributos y recursos patrimoniales que son puestos en valor por el programa para el "consumo turístico" por parte de aquellos grupos sociales para quienes la experiencia de "encuentro" con la historia, la naturaleza y las prácticas autóctonas constituyen referentes de disfrute por motivaciones y significaciones muy diversas. 
En las líneas que siguen se plasma una semblanza general del PPM y de algunos de sus efectos, con base en las evidencias empíricas de que se dispone producto de investigaciones en las que la autora de este texto ha colaborado y de reflexiones con colegas participantes en las mismas, en diferentes localidades en las que ha operado el programa en mención.

Se parte de la presentación de algunas de sus características más relevantes, para centrarse posteriormente en la identificación de lo que se consideran como sus luces y sombras, tomando en cuenta algunos de sus resultados más relevantes, a juicio de la autora. Hay que aclarar, por supuesto, que los límites que imponen los criterios de extensión de una nota crítica no permitirán abordar la reflexión del PPM, agotando el conjunto de las diversas fuerzas y realidades en él implicadas y las múltiples aristas y enfoques desde los que se le puede apreciar analíticamente.

\section{El PPM en contexto}

El PPM se crea en el año 2000 bajo el gobierno de Vicente Fox Quesada y entra en operaciones un año después, con el nombramiento de los dos primeros Pueblos Mágicos (PM): Huasca de Ocampo (en el estado de Hidalgo) y Real del Catorce (en San Luis Potosí). Para el año 2012 los nombramientos se incrementan significativamente a lo largo del territorio nacional, llegando a 83. En 2013 se realiza una revisión del PPM y en 2014 se anuncia la posibilidad de incorporación de nuevas localidades al programa, lo que se concreta en los siguientes cuatro años, llegando a 121 durante el período de Enrique Peña Nieto, ampliándose a 132 en el transcurso del último año de su gobierno. Según información reciente de la propia Sectur (2020a), se reporta la existencia del mismo número de PM actualmente, lo que supone que estos hayan renovado y conservado su nombramiento con base en el cumplimiento de las Reglas de Operación (RO) del programa.

Sin embargo, hay que hacer notar que si bien el espíritu del PPM se ha mantenido a lo largo de los dos períodos de gobierno inmediatamente anteriores al actual y este permanece actualmente como una de las líneas de acción del Programa Nacional de Turismo 2020-2024 (Sectur, 2020b), le han sido incorporados algunos referentes significativos enmarcados en la ruta de la denominada Cuarta Transformación, que constituye el sello material y simbólico del gobierno de López Obrador (2018-2024). 
Estos elementos son visibles en el contenido del propio Plan Sectorial de Turismo (Sectur, 2020b), derivado del actual Plan Nacional de Desarrollo 2019-2024 y de la reciente propuesta de la Estrategia Nacional de PM, publicada en el Diario Oficial de la Federación (DOF, 2020), donde se hace explícita la consideración de que la política derivada de administraciones anteriores se caracterizó por la concentración de los servicios turísticos y la infraestructura "debilitando el pacto social con las mexicanas y los mexicanos, provocando limitantes para un desarrollo económico y social equilibrado entre regiones, y generando la convivencia de paraísos turísticos con zonas de alta marginación" (Sectur, 2020b, párr. 23).

Los nuevos elementos que se incorporan al PPM, se encuentran vinculados a la filosofía social y tanto sus objetivos como el curso de las acciones públicas se sustentan en una noción de desarrollo diferente: “...el turismo tendrá un significado distinto en el que prevalecerán los intereses de la sociedad y se buscará alcanzar la máxima potencialidad de la actividad turística en todas las regiones del país (...) El desarrollo del turismo en México se enfocará en una visión sostenible que logre la protección del patrimonio nacional en cuya esencia está nuestra historia e identidad pluricultural y natural" (Sectur, 2020b, párr. 26).

No obstante, en los documentos rectores del PPM se mantiene la descripción de los PM, identificándolos como aquellas localidades poseedoras de una serie de "atributos simbólicos, leyendas, historia, hechos trascendentes, cotidianidad, en fin, magia que emana en cada una de sus manifestaciones socioculturales, y que significan hoy día una gran oportunidad para el aprovechamiento turístico" (Sectur, 2014, párr. 1).

Asimismo, tal y como ocurría en períodos anteriores, el acceso al PPM supone el cumplimiento de un conjunto de condiciones y criterios diferenciados según distintos momentos (registro, incorporación y permanencia), a los que también se han integrado nuevos elementos vinculados a la perspectiva de los derechos humanos, la equidad, cobertura nacional de desarrollo con equilibrio, recuperación y conservación de recursos patrimoniales, respeto a la diversidad cultural y sustentabilidad, entre los más significativos.

Tales criterios se encuentran contenidos en el documento oficial de las RO, que constituyen las directrices para la puesta en marcha del PPM con el propósito de orientar sus acciones, de manera uniforme, en cada una de las localidades donde opera, a efecto de favorecer la reactivación económica de las localidades mediante la creación de condiciones para el impulso, desarrollo y sustentabilidad del turismo cultural. 
La actual Estrategia Nacional de PM (DOF, 2020) surge de un diagnóstico del PPM en conjunto, considerando algunos de sus efectos y las propias RO con las que operaba. Se parte del análisis de la ineficiencia de los recursos públicos destinados ${ }^{1} \mathrm{du}$ rante el período comprendido entre 2001 y 2018 y la no cobertura de los objetivos, si se toman en cuenta los altos niveles de carencias sociales documentadas por el INEGI y el Consejo Nacional de Evaluación de la Política de Desarrollo Social (Coneval), así como la ausencia de información turística en la que se constaten los logros obtenidos por el PPM.

Igualmente, se señala el no cumplimiento a cabalidad de los lineamientos de permanencia, el bajo nivel de participación de los municipios en el programa, en virtud de que menos del $30 \%$ de ellos cuentan con alguna empresa con certificación turística vigente, la ausencia de criterios que garanticen la composición ciudadana de los Comités de PM, que constituye en teoría una de las figuras fundamentales de participación ciudadana del PPM en los entornos locales², entre otros argumentos.

Asimismo, se alude a los estudios realizados por diversos investigadores que tuvieron como punto de mira en sus investigaciones a los PM, para señalar que estos, además de reconocer el impulso que ha tenido la actividad turística en las comunidades receptoras, se han referido a las deficiencias del PPM para cumplir con el objetivo de fomentar el turismo sostenible y al hecho de que las condiciones de vida de la población local no hayan mostrado mejoría durante la operación del programa, así como a la evidencia de prácticas turísticas que comprometen severamente la preservación del patrimonio.

Con base en tal diagnóstico, se estructura en parte la visión contenida en la nueva Estrategia Nacional de Pueblos Mágicos que se apega a la consolidación del turismo como "un pilar para la economía nacional y un factor prioritario para el bienestar de los mexicanos que viven y trabajan en los entornos turísticos (...) que logre el desarrollo equitativo y justo entre comunidades y regiones, mediante el aprovechamiento sostenible del patrimonio cultural y natural" (DOF, 2020, párr. 74).

\footnotetext{
1 Se indica que, de un monto cercano a los seis mil millones de pesos, el $49 \%$ correspondió a fondos federales y que el $99 \%$ del monto total de los recursos se aplicó al desarrollo de infraestructura y servicios, "dejando en segundo término la innovación, capacitación y calidad con el ejercicio de solo el 1\% de la inversión" a este tipo de acciones (Martínez, 2020, párr. 10).

2 Al respecto, en el diagnóstico en que se respalda y fundamenta la nueva Estrategia Nacional de PM, en relación con estos Comités, se indica que Sectur detectó la deficiencia en los mecanismos para el seguimiento de los acuerdos derivados de las sesiones realizadas por el comité, reflejando la falta de articulación entre el sector público y privado (DOF, 2020).
} 
Esta nueva visión también incorpora la necesaria transformación del turismo para traducirse en una "herramienta de reconciliación social" que favorezca la enmienda del tejido comunitario, de tal manera que se configure en un mecanismo para la "conservación de usos, costumbres, territorios, biodiversidad, identidad, cultura, lengua y legado de los pueblos" que hagan posible el logro del reto de promover transformaciones en el desarrollo turístico, referidas sobre todo al logro de un desarrollo turístico que garantice el equilibrio entre el ámbito local y regional, así como la ampliación de la infraestructura y los servicios a más destinos (DOF, 2020, párr. 78).

\section{Luces y sombras del programa}

EI PPM como figura del turismo cultural en México que pretende articular la dimensión local de las entidades donde opera, incorporando en su oferta turística el conocimiento (y disfrute) de la experiencia de "encuentro" con la otredad, así como sus símbolos materiales y simbólicos condensados en el patrimonio, ha implicado luces y sombras. Esto es, aspectos positivos y negativos derivados de elementos relacionados con su operación en el curso de su puesta en marcha.

Tomando en consideración la información de que se dispone, producto de la participación de la autora de esta nota crítica en investigaciones orientadas al conocimiento de su operación y efectos sociales, se harán algunas consideraciones al respecto. En las apreciaciones, se hace énfasis en las evidencias encontradas en el pueblo mágico de El Fuerte, en Sinaloa, y en los de Álamos y Magdalena de Kino en el estado de Sonora, muchas de las cuales son coincidentes, como se observa en los trabajos de Enríquez y Guillén (2017); Enríquez et al. (2015a); Enríquez et al. (2015b) y Enríquez et al. (2016).

Entre las denominadas luces hay que señalar sin duda los beneficios del PPM a nivel local, derivados de la diversificación de sus fuentes de derrama económica gracias a la actividad turística que amplió su espectro de visibilidad nacional y en muchos casos internacional, entre otros beneficios.

Este elemento positivo coincide con el análisis realizado por diversos especialistas sobre la presencia y características del turismo cultural, acudiendo en este momento a dos de ellos, precisamente por sus referencias al papel central que cumple este enfoque de impulso y desarrollo turístico. 
Se recupera a Ana Mantecón (2010) cuando se refiere a que el turismo cultural ha significado una opción favorable, en el contexto de su transformación como una de las mayores industrias del mundo, por lo que podría hablarse de una industria del patrimonio. Esto ha favorecido sin duda que México se haya convertido en uno de los 15 destinos turísticos de mayor afluencia de visitantes del mundo.

Por su parte, Balslev y Velázquez-García (2013, pág. 11) han planteado que la opción del turismo cultural en México ha recibido atención de los gobiernos en los últimos años, en virtud de considerarla "[...] una respuesta a la baja en la actividad económica de las regiones agrícolas y las pequeñas ciudades [...]", de ahí que programas como el de Pueblos Mágicos hayan destinado sus acciones al logro de este fin, concentrándolas sobre todo a la construcción de infraestructura y al respaldo de aquellas actividades económicas vinculadas al turismo (Balslev y Velázquez-García, 2013).

Necesario referirse también a los beneficios locales del PPM en el mejoramiento de la infraestructura y equipamiento urbanos, así como en la ampliación y diversificación de las actividades económicas vinculadas a los sectores del comercio y los servicios, que sin duda han influido no solo en mejorías urbanas y económicas, sino en la capacidad de atracción de visitantes que favorecen el incremento del consumo en las localidades donde opera el programa.

Por tanto, es posible decir que el ángulo positivo del PPM se centra o hasta ahora en su mayoría se ha centrado en los beneficios operados en la infraestructura y fisonomía de los pueblos, así como en las diversificaciones parcialmente operadas en los giros económicos con el fortalecimiento del comercio y los servicios; sin embargo, desde luego que estos no se han reflejado hasta ahora en beneficios para la población en su conjunto, por lo que, si se atienden los objetivos del PPM, puede decirse que estos solo se han logrado muy parcialmente.

Algunos de los aspectos que se han tomado en cuenta para identificar las sombras del PPM tienen que ver precisamente con sus beneficios, en términos de las posibilidades de apropiación por la población en su conjunto, si consideramos al objetivo central del mismo que es la reactivación económica de las localidades.

A pesar de que la lógica y el sentido del PPM se orientan al logro de un desarrollo incluyente, la realidad del programa en su dimensión operativa ha derivado en favores para el sector turístico, en este caso para las empresas hoteleras y gastro- 
nómicas; pero, aunque la población local aparece como beneficiaria en el discurso gubernamental, hasta ahora ha tenido resultados positivos marginales de la actividad turística.

Asimismo, en referencia al "consumo" que tiene lugar en los entornos de turismo cultural, como los PM, es importante señalar los efectos de la mercantilización del patrimonio, el cual se ofrece al turista más como un "objeto para el consumo" que como un elemento vinculado al propio entorno sociocultural, como expresión de la singularidad que ofrece sobre la historia y la propia identidad colectiva para el conocimiento y reconocimiento de la otredad en la construcción de este tipo de experiencia turística.

Al respecto, la perspectiva crítica de Prats y Santana (2011) sobre las tendencias actuales del turismo cultural, advierte sobre la condición de la nueva demanda del turismo cultural por parte de los viajeros que ha sido capitalizada por el mercado, de ahí que "cada vez abundan más los destinos que ofrecen productos supuestamente auténticos y singulares, mientras que, por otra parte, su potencialidad ha permitido reconvertir o crear ex novo una nueva oferta de productos y destinos, normalmente de interior o en todo caso en zonas no explotadas o explotadas muy tangencialmente por los grandes operadores turísticos" (Prats y Santana, 2011, pág. 10).

Por lo que respecta a las expectativas de la población local en relación con el PPM, de acuerdo con las evidencias de investigación de este trabajo puede afirmarse que existen dificultades y obstáculos derivados de lo que la comunidad quiere e imagina de su pueblo, en comparación con lo pretendido por las autoridades municipales y de turismo en la promoción y oferta turística de la localidad. Diversas luchas simbólicas afloran en el imaginario social en términos de la existencia de ganadores y perdedores dentro del proceso de turistificación de la localidad.

Entre los actores del turismo cultural, por ejemplo, tienen especial protagonismo aquellos que son actores productores en las entidades de servicios, referidos fundamentalmente a restauranteros y hoteleros, así como a los propios guías turísticos que se ocupan de conducir a los visitantes por los espacios y contornos de los PM.

No obstante, encontramos por una parte también a un conjunto de vendedores ambulantes con escasa "visibilidad" para el turista, en virtud de haber sido desplazados del primer cuadro de las ciudades, de acuerdo con el requerimiento de las 
propias RO que señalan que únicamente pueden realizar el comercio ambulante quienes ofrezcan productos locales representativos de las comunidades.

De igual manera, en la mayoría de los casos las relaciones entre anfitriones y turistas están desdibujadas, gracias a las distancias simbólicas entre la diversidad de actores productores. Los pequeños comerciantes y quienes se dedican al comercio ambulante, por ejemplo, consideran que quienes se benefician del turismo son los hoteleros y restauranteros de mayor calado.

Igualmente, en lo que respecta al flujo de visitantes y a las características y posibilidades de la "autenticidad" de la experiencia turística en los PM, se ha encontrado que la experiencia del turista suele estar ya predeterminada por el conocimiento previo que tienen entre sí anfitriones y turistas, a través de mecanismos electrónicos y virtualizados, al contratar los servicios por medio de la información en red, sobre todo en el caso del turismo internacional, aunque también ocurre con el turismo local.

Esto implica, entre otras cosas, que la imagen reconocida previamente sea la que se busca encontrar en las localidades y que las posibilidades de adentrarse en el conocimiento de usos y costumbres, prácticas y expresiones culturales locales se reduzca y se limite, con una derrama económica que beneficie a mayores segmentos de la población.

Sin embargo, es menester señalar que, de acuerdo con las propias observaciones de la autora de esta nota crítica, las prácticas de los visitantes en estos entornos turistificados a partir de la operación del PPM se encuentran diferenciadas entre turistas internacionales y turistas locales: los primeros suelen tener una ruta establecida en forma predeterminada por el empresario hotelero y quien operará como guía, mientras que los segundos tienen la posibilidad de establecer vínculos más directos con los anfitriones y tienen mayor libertad de movilidad en el entorno del pueblo mágico y sus alrededores, así como mayor contacto e intercambios con pequeños comerciantes establecidos y los ambulantes.

Resulta importante también señalar entre las sombras del PPM los efectos negativos que produce en algunos pobladores locales, si se atiende a la molestia que suele apreciarse en algunas representaciones locales de la actividad turística que ha sido expresada en forma explícita, referida al turismo en lo general como actividad 
que beneficia a un sector muy localizado (empresas restauranteras y hoteleras) y no al pueblo en su conjunto.

Esta situación concuerda con uno de los planteamientos de Muñoz (2017), cuando considera que la prioridad de este programa ha sido apoyar y desarrollar la industria del turismo con miras a satisfacer las necesidades de los visitantes y no así el impulso del turismo como un medio para el desarrollo sustentable de los poblados adscritos al mismo. La autora añade que mientras la meta de las Reglas de Operación en curso es fomentar la obra pública y otras acciones a fin de desarrollar la industria turística, los proyectos abocados a satisfacer las necesidades locales y resolver sus problemáticas, así como al fomento de la valorización del patrimonio, han sido escasos, si no es que nulos.

Para cerrar la reflexión y a manera de corolario en este apartado, hay que decir que el PPM como uno de los emblemas del turismo cultural de los últimos años en México constituye una suerte de "crisol", que refleja en su estructura, operación y efectos, la realidad de diversos enfoques de desarrollo, tramas políticas, esquemas organizativos y distintos grados de madurez en la participación ciudadana.

Hasta ahora los gobiernos que han impulsado el curso de acción del PPM desde el momento de su surgimiento en 2001 y el "clímax" de su cobertura operada a finales de 2018, con 132 localidades beneficiadas con el nombramiento, ha implicado un momento de impasse en la transición política actual con el gobierno de López Obrador. Todavía no existen evidencias concretas suficientes para identificar las diferencias de la nueva Estrategia Nacional de PM propuesta por el gobierno actual, en términos de su puesta en marcha y sus efectos, para estar en condiciones de apreciarlas analíticamente más allá de la identificación ya realizada, con base en el contenido formal (discursivo) del documento rector de la misma.

\section{Referencias}

Balslev, H. y Velázquez-García, M. A. (2013). El turismo cultural en Argentina, México y Brasil. Avances y desafíos. Diálogos Latinoamericanos, 21, 9-30. https://bit.ly/ 3bwykPp

Diario Oficial de la Federación (DOF). (2020, 1 de octubre). Estrategia Nacional de Pueblos Mágicos. https://bit.ly/3qqTGSs 
Enríquez, J. A., Guillén, M., Jaime, M. E. y Valenzuela, B. A. (2015a). Imaginarios sociales de la comunidad en el pueblo mágico de El fuerte, Sinaloa, México. Imagonautas, 5, 82-99. https://bit.ly/2PQ1X50

Enríquez, J., Guillén, M., Valenzuela, B. y Jaime, M. (2015b). Magdalena de Kino, Sonora. Percepciones de la población con respecto al patrimonio cultural y el turismo. Topofilia, Revista de Arquitectura, Urbanismo y Ciencias Sociales, 5(1), 67-88. https://bit.ly/3t9onNR

Enríquez, J., Guillén, M., Valenzuela, B. y Jaime, M. (2016). Rediseño de ciudades turísticas en el noroeste de México. QARTUPPI. https://bit.ly/3qsHHUh

Enríquez, J. A. y Guillén, M. (2017). Turismo cultural e identidad en el pueblo mágico de Álamos, Sonora. International Journal of Scientific Management and Tourism, 3(4), 495-512. https://bit.ly/3eoiulv

Instituto Nacional de Estadística y Geografía (INEGI). (2019). Estadísticas a propósito del día mundial del turismo. https://bit.ly/3qr5LHi

Mantecón, A. (2010). El giro hacia el turismo cultural: Participación comunitaria y desarrollo sustentable. Cuadernos No. 20. Patrimonio Cultural y Turismo, 107-130. https://bit.ly/3qsopi5

Martínez, A. (2020, 1 de octubre). Solo 16 de 121 Pueblos Mágicos cumplen con los requisitos del programa: Sectur. La Razón. https://bit.ly/3ciouzz

Muñoz, E. (2017). La puesta en valor del patrimonio cultural como oferta turística mediante el programa Pueblos Mágicos ¿Una alternativa para fortalecer las culturas locales o para su fragmentación? Pensamiento y acción cultural para la paz y la participación ciudadana [ponencia]. $2^{\circ}$ Congreso Latinoamericano de Gestión Cultural, Cali, Colombia.

Prats, L. y Santana, A. (Coords.). (2011). Turismo y patrimonio, entramados narrativos. Colección PASOS Edita No. 5. https://bit.ly/2OD9jJc

Secretaría de Turismo (Sectur). (2014). Guía de incorporación y permanencia Pueblos Mágicos. https://bit.ly/2PKB2br

Secretaría de Turismo (Sectur). (2020a, 1 de diciembre). Pueblos Mágicos de México. https://bit.ly/3ckPoIP

Secretaría de Turismo (Sectur). (2020b). Programa sectorial de turismo 2020-2024. https://bit.ly/3ev52Tf 